\title{
The Impact of Financial Agglomeration on Regional Economic Growth Xie Chunqing
}

\author{
School of Economics and Commerce South China University of \\ Technology,Guangzhou,Guangdong Province,China
}

xie_chunqing@126.com

\begin{abstract}
Keywords: Regional economic growth, Financial agglomeration, Information flow, Financial innovation
\end{abstract}

\begin{abstract}
Based on the re-deduction of endogenous economic growth theory, this paper analyzes the mechanism of financial agglomeration affecting regional economic growth from the perspective of information flow and financial innovation, and proposes two theoretical hypotheses accordingly. Finally, this paper selects the data from 2004 to 2015 in 31 provinces of China as the research sample, and makes the regression analysis by GMM method. We finds that all the variables proposed in this paper have passed the $1 \%$ significance level hypothesis test, but only the cross-term of financial agglomeration and information hinterland border $(\mathrm{FA} * \mathrm{IH})$ and the cross-term of financial agglomeration and financial innovation $(\mathrm{FA} * \mathrm{FI})$ are positive, which is consistent with the theoretical hypothesis proposed in this paper.
\end{abstract}

\section{金融集聚对区域经济增长的影响研究}

\author{
谢春清 \\ 华南理工大学经济与贸易学院, 广州, 广东, 中国 \\ xie_chunqing@126.com
}

关键词：区域经济增长，金融集聚，信息流，金融创新

中文摘要. 本文基于内生经济增长理论的重新推导基础上，从信息流视角和金融创新视角分 析金融集聚程度影响区域经济增长的机制，并依此提出两个理论假设。最后本文选取我国31 个省份2004-2015年的数据为样本, 通过动态面板广义矩估计 (GMM) 方法进行回归分析, 本文提出的所有变量均通过了 $1 \%$ 显著性水平假设检验，但只有金融集聚程度与信息腹地边界 的交叉项（FA*IH）和金融集聚程度与金融创新的交叉项（FA*FI）的数估计值为正，符合本 文提出的理论假设。

\section{1、引言}

18至19世纪，在以Adam Smith和Ricardo为代表提出的古典经济增长理论中，认为货币对 经济增长的作用是中性的。而Schumpeter(1911) ${ }^{[1]}$ 认为金融中介提供的服务体现在五个方面: 动员储蓄、评估项目、管理风险、监督管理者和促进交易, 这些服务对技术创新和经济发展 是至关重要的。

自Goldsmith（1969）针对35个国家从1860年至1963年的跨国数据进行实证研究，采用金 融中介的资产与GNP的比重表示金融发展水平，发现经济增长和金融发展存在密切关系之后 [2], 学者们开始重视金融在经济增长中发挥的重要作用, 并取得了众多实证研究成果。 King\&Levine (1993) 同样通过实证分析, 发现金融负债规模、金融机构类别、金融资产分布 等衡量金融发展水平的指标和经济增长指标呈现显著正相关，该结果证明Schumpeter(1911) 
的观点是正确的: 金融可以促进经济增长 ${ }^{[3]}$ 。Levine $(1997 ; 2005)^{[4,5]}$ 认为金融体系对经济增长 的作用主要体现在风险管理、储蓄聚集、资本配置、公司治理、以及商品和金融合同交易等 方面,而最终都是通过“资本积累”和“技术创新”这两条途径来实现的。

现有的上述研究成果从不同视角验证了金融发展对经济增长的正向影响作用，但这些研 究成果均倾向于将区域作为封闭的整体，忽略了金融机构和金融资源的流动性。从 20 世纪 80 年代开始，随着经济一体化和金融全球化的发展，金融资源在区域间的流动加速，金融机构 开始在少数几个中心城市聚集起来，金融集群已经成为现代金融产业组织的基本形式 ${ }^{66]}$ 。关 春燕（2013）和许哲（2015）认为金融集聚提高了金融服务的专业化程度从而提高金融服务

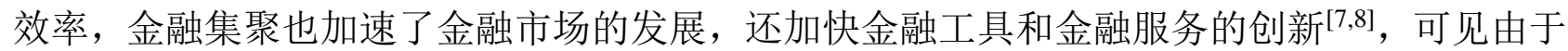
金融机构空间运动而形成的金融集聚已经改变了金融规模、金融市场、金融工具等方面的金 融发展指标。因此研究金融集聚对区域经济增长的影响是很有必要的。

目前关于金融集聚影响经济增长的研究成果主要是国内文献。刘军等（2007）[9]从理论 上分析金融集聚影响经济增长的机制, 认为金融集聚主要通过金融集聚效应、金融扩散效应 和金融功能促进实体经济增长。陆军和徐杰（2014） ${ }^{[10]}$ 以京津冀10个城市为样本进行实证分 析, 研究发现:受北京经济发展影响, 北京的金融集聚得到不断强化, 对天津、河北地区产生 扩散效应，对周边地区经济发展具有拉动作用。

本文将在前人研究的基础上从信息流视角和金融创新视角探讨金融集聚影响经济增长的 机制, 并提出理论假设, 通过实证方法论证理论假设是否成立, 从而揭露金融集聚影响区域 经济增长机制的本质。

\section{2、理论分析}

\section{1 内生经济增长理论}

本文首先从内生经济增长理论出发，来探寻金融产业集聚促进区域经济增长的理论基础。 Romer(1987)、Rebero (1987，1991)、Barro（1991)、Benhabib\&Jovanovic（1991）等提出的 $\mathrm{AK}$ 模型, 最简单也最合理地解释了金融发展影响经济增长的渠道; 此外, 我国蔡昉和都阳 (2000)、林毅夫和刘培林(2003)等学者经过实证检验发现, 资本积累是中国经济增长的主要驱 动力, 也就是说模型能够描述中国的经济增长。

Pagano（1993） [11]在 AK 模型基础上研究金融发展影响经济增长的可能渠道。在储蓄率 不变和技术水平既定的假定条件下，生产函数是资本存量的线性函数:

$$
Y_{t}=A K_{t}
$$

其中, $\mathrm{Y}$ 为该地区的 GDP, $\mathrm{A}$ 为资本的边际生产率; $\mathrm{K}$ 为资本存量。投资 $\mathrm{I}$ 可由式 (2) 表示:

$$
I_{t}=K_{t+1}-(1-\delta) K_{t} \Rightarrow K_{t+1}-K_{t}=I_{t}-\delta K_{t}
$$

其中, $\mathrm{I}$ 为投资， $\delta$ 表示资本的折旧率。在均衡条件下，关于经济增长率有如下公式:

$$
\Delta Y_{\mathrm{t}+1}=Y_{t+1}-Y_{t}=A\left(K_{t+1}-K_{t}\right)
$$

将式（2）代入式 (3), 可得:

$$
\Delta Y_{t+1}=A\left(I_{t}-\delta K_{t}\right)=A I_{t}-\delta Y_{t}
$$

又因为储蓄和投资的关系如下:

$$
I_{t}=\theta S_{t}=\theta * S_{t} * Y_{t}
$$

其中, $\mathrm{S}$ 为储蓄, $\mathrm{s}$ 为储蓄率即储蓄占总产出的比重, 为储蓄向投资转化的比例。将式 （4）和式 (5) 代入经济增长率的公式, 则 $(t+1)$ 期经济增长率为:

$$
g_{t+1}=\frac{\Delta Y_{t+1}}{Y_{t}}=\frac{A I_{t}-\delta Y_{t}}{Y_{t}}=\frac{A \theta S_{t}}{Y_{t}}-\delta=A \theta s_{t}-\delta
$$

其中, $\mathrm{g}$ 表示经济增长率。 
由式 (6) 可以看出, 影响经济增长率的因素有四个: 资本边际生产率 A、私人储蓄率 $\mathrm{s} 、$ 储蓄向投资的转化率、资本折旧率。金融集聚通过影响资本边际生产率 $\mathrm{A}$ 、私人储蓄率 $\mathrm{s}$ 、 储蓄向投资的转化率，进而影响经济增长率。

\section{2信息流视角下金融集聚影响经济增长的机制分析}

金融地理学（Financial Goegraphy）首次将信息的作用应用到金融领域中，为研究金融问 题提供了一个新的视角。金融中心提供专业及高附加值的中介服务的, 很大程度上依存于信 息。信息与金融中心唇齿相依，前者主导着后者发展的步伐和方向。简言之，金融业也可被 喻为 “高增值”的信息服务业（Porteous,1995,1999;Martin,1999;Thrift,1994）[12-15]。

赵晓斌等 (2002) [16]将信息分为标准化信息和非标准化信息: 标准化信息是可被媒体传 送和散布的 “硬” 资料,其传播质量几乎不受距离的影响; 非标准化信息则是不可被媒体如实 传送的 “软” 资料(如谣言、公司负债消息等), 其传播质量明显受到地理距离和社会距离的 影响。传递非标准化信息很可能会引致信息诠释的误差,从而影响到整个商业决策。Thrift (1994) [15]指出, 非标准化信息的传递与获得具有歧视性和边际成本递增性, 要想准确利用非标准化 信息的价值，最好的办法就是尽可能接近信息源。在全球化经济体系中，由于金融市场日趋 投机，金融机构应该进驻信息腹地，凭借 “地方” 信息辨析市场。当金融机构为了冲破地理 约束，大规模集中到信息源时，金融中心便水到渠成。

金融机构集聚会带来人才的集聚，金融人才是非标准化信息的最佳携带者和传播者。集 聚在同一个区域内的金融机构、会计师事务所等其他中介机构以及企业之间为了合作或竞争 而频繁的互动会形成错综复杂的集群网络, 该集群网络为信息流在集聚区内的高速有效流动 提供了多样化的渠道。

综上，金融机构集聚带来了信息流的集聚，尤其是非编码信息流的集聚，而通过网络效 应, 信息流效应得到加强, 使得信息流能高效快速地在集聚区内流动, 从而创造价值。信息 流的快速传播提高了金融效率，降低了企业和金融机构以及会计师事务所等其他中介机构之 间的交易成本和交易信息搜寻费用，实际上信息流有利于资金投向高生产率的实体部门，从 而提高了资本的边际生产率 $\mathrm{A}$ 。信息流效应还提高了投资者和筹资者的连接速度，尤其为私 人投资者提供了更多的投资机会，从而提高了私人储蓄率 $\mathrm{s}$, 提高了储蓄向投资的转化率。根 据本章第一节推导的内生经济增长理论可知, 资本边际生产率、储蓄率、储蓄向投资转化率 的提高，增加了资本积累和提高技术创新，从而提高经济增长率。

基于以上的理论分析，本文提出第一个理论假设 $\mathrm{H} 1$ ：金融集聚会带来信息流集聚，信息 流效应通过降低企业的交易成本和信息搜寻成本，从而促进经济增长。

\section{3金融创新视角下金融集聚影响经济增长的机制分析}

金融集聚区内由于金融机构密集度较大，金融集聚不可避免的加大竞争激烈程度，而竞 争压力也是区域金融创新的一种动力。由于金融集聚区金融机构之间激烈竞争，为了满足客 户需求, 并在竞争中发展, 金融机构在金融 “需求追求” 与 “供给领先” 的动力下, 不断进 行金融创新。金融机构进行各种金融产品和服务的创新, 金融创新产品一经推出, 会被竞争 对手学习模仿, 创新产品与服务逐渐外溢到区内的其它相关机构。所以, 金融机构的竞争比 其它分散区域的金融机构间的竞争更激烈，金融集聚区金融机构的竞争优势更易于进入金融 业最前沿。另外，由于金融机构的地理临近性，同行业之间的工作人员有着大量的非正式接 触与交流，使得创新思想不断交流，隐性知识不断被激发，加快了金融创新速度。所以，集 聚区金融机构的竞争与合作所产生的创新效应是金融集聚效应促进城市竞争力提升的重要方 面。

综上，金融集聚通过知识溢出效应和区域创新网络效应提高区域金融创新速度，金融创 新包括金融产品创新和金融服务创新。金融创新提高了资本的边际生产率 $\mathrm{A}$ 、私人储蓄率 $\mathrm{s}$ 和储蓄向投资的转化率，从而促进经济增长。

基于本小节的理论分析，本文提出第二个理论假设 H2：金融集聚通过知识溢出效应和区 
域创新网络效应提高区域金融创新速度, 金融创新提高了资本的边际生产率、私人储蓄率和 储蓄向投资的转化率，从而促进经济增长。

\section{3.实证分析}

\section{1金融集聚程度测量}

本文选取金融业从业人数区位商、金融业增加值区位商、金融机构存款余额区位商、金 融机构贷款余额区位熵、保费收入区位商、证券市场交易额区位熵这六个指标，运用因子分 析法计算代表金融集聚程度的综合因子得分。

本文以我国 31 个省份为研究样本，选取 2004-2015 年的区间长度，在此基础上进行的实 证分析。其中，金融业增加值、金融业从业人数、金融机构存款余额、金融机构贷款余额、 保费收入等数据来源于《中国统计年鉴》、《中国城市统计年鉴》、《国民经济和社会发展统计 公报》; 证券市场交易额的数据来源于《深圳证券交易所市场统计年鉴》、《上海证券交易所统 计年鉴》。

本文通过 SPSS17.0 计算代表各省份金融集聚程度的综合因子得分 FA。首先，对数据进 行 “0-1” 标准化处理; 第二步, KMO 检验和 Bartlett(1937)球形度检验, 从表 1 可以看出 KMO 值较大且 Bartlett 检验结果非常显著, 因此本文的数据适合做因子分析。

表1＼cjkstart因子分析KMO值和Bartlett检验结果

\begin{tabular}{l|l|r}
\hline \multicolumn{2}{|l|}{ KMO 检验结果 } & 0.849 \\
\hline \multirow{4}{*}{ Bartlett 的球形度检验结果 } & 近似卡方 & 5172.662 \\
\cline { 2 - 3 } & 自由度 & 15 \\
\cline { 2 - 3 } & 显著性程度 $(\mathrm{p}$ 值 $)$ & 0.000 \\
\hline
\end{tabular}

第三步，选取公因子。根据初始特征根大于等于 1 的原则，选取一个公因子，其累积贡 献率达到 $86.11 \%$ (见表 3)。

表2 因子分析的解释总方差

\begin{tabular}{l|r|r|r|r|r|r}
\hline & \multicolumn{1}{|l|}{ Factor1 } & Factor2 & \multicolumn{1}{|l|}{ Factor3 } & \multicolumn{1}{|l}{ Factor4 } & \multicolumn{1}{l|}{ Factor5 } & \multicolumn{1}{l}{ Factor6 } \\
\hline 特征值 & 5.167 & 0.584 & 0.193 & 0.045 & 0.006 & 0.005 \\
\hline 比重 & 0.861 & 0.097 & 0.032 & 0.007 & 0.001 & 0.001 \\
\hline 累积 & 0.861 & 0.959 & 0.991 & 0.998 & 0.999 & 1.000 \\
\hline
\end{tabular}

第四步, 计算综合因子得分。依据各变量的因子得分系数 (见表 3), 将因子得分系数与 对应因子相乘后相加得出本文的金融集聚程度。部分省份的金融集聚程度如表 4 所示。

表3 因子分析的各变量因子得分系数

\begin{tabular}{l|r}
\hline \multicolumn{1}{c|}{ 变量 } & \multicolumn{1}{|c}{ 因子得分系数 } \\
\hline 金融业增加值区位熵（FV） & 0.156 \\
\hline 金融机构存款区位商（FD） & 0.192 \\
\hline 金融机构贷款区位熵（FL） & 0.190 \\
\hline 保费收入（PI） & 0.190 \\
\hline 证券市场交易额（ST） & 0.179 \\
\hline 金融业从业人数区位熵（FE） & 0.168 \\
\hline
\end{tabular}


表4＼cjkstart全国部分省份2004-2015年金融集聚程度

\begin{tabular}{l|cccccccccccc}
\hline \multicolumn{1}{c}{ 年份 } & $\mathbf{2 0 0 4}$ & $\mathbf{2 0 0 5}$ & $\mathbf{2 0 0 6}$ & $\mathbf{2 0 0 7}$ & $\mathbf{2 0 0 8}$ & $\mathbf{2 0 0 9}$ & $\mathbf{2 0 1 0}$ & $\mathbf{2 0 1 1}$ & $\mathbf{2 0 1 2}$ & $\mathbf{2 0 1 3}$ & $\mathbf{2 0 1 4}$ & $\mathbf{2 0 1 5}$ \\
\hline 广西 & 0.03 & 0.03 & 0.03 & 0.03 & 0.04 & 0.04 & 0.03 & 0.03 & 0.03 & 0.03 & 0.03 & 0.03 \\
\hline 内蒙古 & 0.05 & 0.05 & 0.05 & 0.04 & 0.04 & 0.05 & 0.05 & 0.04 & 0.04 & 0.04 & 0.04 & 0.04 \\
\hline 吉林 & 0.07 & 0.07 & 0.06 & 0.05 & 0.05 & 0.05 & 0.04 & 0.04 & 0.04 & 0.04 & 0.04 & 0.04 \\
\hline 黑龙江 & 0.04 & 0.04 & 0.05 & 0.06 & 0.05 & 0.05 & 0.05 & 0.05 & 0.06 & 0.06 & 0.06 & 0.06 \\
\hline 重庆 & 0.09 & 0.09 & 0.09 & 0.08 & 0.08 & 0.08 & 0.08 & 0.09 & 0.10 & 0.09 & 0.09 & 0.08 \\
\hline 广东 & 0.10 & 0.09 & 0.09 & 0.10 & 0.10 & 0.10 & 0.09 & 0.09 & 0.09 & 0.09 & 0.09 & 0.09 \\
\hline 浙江 & 0.11 & 0.12 & 0.12 & 0.12 & 0.13 & 0.13 & 0.13 & 0.13 & 0.12 & 0.11 & 0.10 & 0.10 \\
\hline 天津 & 0.18 & 0.18 & 0.20 & 0.20 & 0.19 & 0.19 & 0.19 & 0.19 & 0.19 & 0.19 & 0.19 & 0.20 \\
\hline 北京 & 0.46 & 0.51 & 0.49 & 0.47 & 0.45 & 0.44 & 0.44 & 0.44 & 0.46 & 0.43 & 0.44 & 0.45 \\
\hline 上海 & 0.79 & 0.81 & 0.80 & 0.79 & 0.74 & 0.71 & 0.70 & 0.70 & 0.71 & 0.68 & 0.65 & 0.65 \\
\hline
\end{tabular}

\section{2 样本与数据}

本文选取 GDP 的增长率作为因变量 EG 的指标; 采用因子分析法计算的综合因子得分表示 金融集聚程度 (FA); 本文引入的两个交叉项分别为信息流与金融集聚程度的乘积、金融创新 与金融集聚程度的乘积。其中，本文将参考 Zhao (2003) 对信息腹地边界和信息厚度的定义， 认为信息腹地是是信息流的最佳访问点, 而信息腹地边界是该最佳访问点对周边地区的辐射 和影响的最大范围, 信息厚度即衡量信息流的量的大小 ${ }^{[17]}$, 因此本文选取旅客周转量指标代 表信息腹地边界 (IH), 邮电业务总量指标代表信息厚度 (IT), 从这两个角度衡量信息流 (IF); 根据蒋岳祥和蒋瑞波（2013）的观点，本文选择其他金融资产与现金形式资产之间的比率作 为区域金融创新能力 (FI) 的衡量指标，其中，其他金融资产包括当年国内股票筹资和当年国 内债券筹资总额，现金形式资产包括银行贷款余额。依据内生经济增长理论模型，影响经济 增长的最重要的两个因素就是资本积累和劳动力, 因此本文实证模型中加入的实物资本 (PC) 和人力资本 $(\mathrm{HC})$ 作为控制变量。以上所有数据来源于 《区域金融运行报告》、《中国城市统 计年鉴》和《国民经济和社会发展公报》。

变量描述性统计分析如表 5 所示。

表5 变量描述性统计分析

\begin{tabular}{l|c|c|r|r|r|r|r}
\hline \multicolumn{1}{c|}{ 变量名称 } & 简称 & 指标 & 样本量 & 均值 & 标准差 & \multicolumn{1}{c|}{ 极小值 } & \multicolumn{1}{c}{ 极大值 } \\
\hline 经济增长 & $\mathrm{EG}$ & GDP 增长率/\% & 372 & 15.75 & 7.65 & -0.68 & 62.71 \\
\hline 金融集聚程度 & $\mathrm{FA}$ & 综合因子得分 & 372 & 0.09 & 0.14 & 0.02 & 0.81 \\
\hline 金融创新 & $\mathrm{FI}$ & $\begin{array}{c}\text { 其他形式金融资 } \\
\text { 产/现金形式金融 } \\
\text { 资产 } / \%\end{array}$ & 372 & 14.50 & 11.06 & 0.00 & 70.10 \\
\hline 信息腹地边界 & $\mathrm{IH}$ & $\begin{array}{c}\text { 旅客周转量/亿人 } \\
\text { 公里 }\end{array}$ & 372 & 867.23 & 677.69 & 7.00 & 4852.00 \\
\hline 信息厚度 & $\mathrm{IT}$ & $\begin{array}{c}\text { 邮电业务总量/亿 } \\
\text { 元 }\end{array}$ & 372 & 1108.05 & 1365.14 & 13.47 & 13251.26 \\
\hline 实物资本 & $\mathrm{PC}$ & $\begin{array}{c}\text { 全社会固定资产 } \\
\text { 投资额/亿元 }\end{array}$ & 372 & 8756.25 & 8423.93 & 168.44 & 48312.46 \\
\hline 人力资本 & $\mathrm{HC}$ & $\begin{array}{c}\text { 年平均就业人数 } \\
\text { 总数/万人 }\end{array}$ & 372 & 2466.88 & 1711.29 & 135.00 & 6711.00 \\
\hline
\end{tabular}

单位根检验。为了消除量纲的影响，便于不同变量之间进行比较，本文首先对实证模型 中涉及的变量全部进行“ $0-1$ ”标准化处理, 在此基础上进行以下的检验和回归。本文分别选取 LLC、IPS、ADF、PP 作为单位根检验指标。从表 6 的单位根检验结果来看, 原始数据中 EG、 FA、FI 和 $\mathrm{HC}$ 在 $1 \%$ 的显著性水平拒绝了原假设, 即这四个变量的原始序列是平稳序列。而 IH、IT、PC 这三个变量在 $10 \%$ 的显著性水平下均无法拒绝原假设, 原始序列均为非平稳序列。 经过一阶差分处理后, 所有变量均在 $1 \%$ 的显著性水平下拒绝了原假设, 即所有变量的一阶差 分序列是平稳序列。 
表6 各变量单位根检验结果

\begin{tabular}{|c|c|c|c|c|c|c|c|c|}
\hline \multirow{2}{*}{$\begin{array}{l}\text { 变量 } \\
\text { 名称 }\end{array}$} & \multicolumn{2}{|c|}{ LLC 检验 } & \multicolumn{2}{|c|}{ IPS 检验 } & \multicolumn{2}{|c|}{ ADF 检验 } & \multicolumn{2}{|c|}{ PP 检验 } \\
\hline & 原始数据 & 一阶差分 & 原始数据 & 一阶差分 & 原始数据 & 一阶差分 & 原始数据 & 一阶差分 \\
\hline EG & $-8.86^{* * *}$ & $24.22^{* * *}$ & $-4.56^{* * *}$ & $-15.02^{* * *}$ & $-6.76^{* * *}$ & $-17.60^{* * *}$ & $-7.25^{* * *}$ & $19.36^{* * *}$ \\
\hline FA & $4.10^{* * *}$ & $15.71^{* * *}$ & $4.47^{* * *}$ & $8.70^{* * *}$ & $1.89^{* * *}$ & $13.14^{* * *}$ & $1.96^{* * *}$ & $13.45^{* * *}$ \\
\hline FI & -0.06 & $-25.01^{* * *}$ & $-6.92^{* * *}$ & $-6.53^{* * *}$ & 1.66 & $-16.49^{* * *}$ & 2.57 & $-17.56^{* * *}$ \\
\hline $\mathrm{IH}$ & 8.62 & $-11.85^{* * *}$ & 1.02 & $-9.24^{* * *}$ & 9.12 & $-10.24^{* * *}$ & 10.40 & $-10.83^{* * *}$ \\
\hline IT & 13.33 & $-2.72^{* * *}$ & 17.03 & $-3.05^{* * *}$ & 20.35 & $-2.97^{* * *}$ & 21.79 & $-3.55^{* * *}$ \\
\hline $\mathrm{PC}$ & 25.32 & $-9.43^{* * *}$ & 3.14 & $-2.54^{* * *}$ & 3.53 & $-3.57^{* * *}$ & 3.72 & $-1.87^{* * *}$ \\
\hline $\mathrm{HC}$ & 16.90 & $-30.76^{* * *}$ & $-9.06^{* * *}$ & $-9.39^{* * *}$ & 14.91 & $-8.04^{* * *}$ & 16.03 & $-6.53^{* * *}$ \\
\hline
\end{tabular}

注: “***” 表示通过 $1 \%$ 显著性水平检验; “**” 表示通过 $5 \%$ 显著性水平检验; “*” 表示通过 $10 \%$ 显著性 水平检验; 以下相同。

协整检验。本文运用 eviews6.0 进行协整检验结果如表 7 所示，Kao 检验结果表明在 1\% 的显著性水平下拒绝协整检验原假设“变量之间不存在协整关系”, 这是一种长期均衡的关系, 即使在短期内变量偏离了它们的关系, 但从长期看它们会恢复到它们原有的联系中去。因此 本文的数据适合做回归分析。

表7 面板数据协整检验结果

\begin{tabular}{|l|l|c|c|}
\hline 检验方法 & 检验统计量 & 统计值 & P 值 \\
\hline Kao 检验 & 面板 $\mathrm{ADF}$ 统计量 & -4.62 & 0.00 \\
\hline
\end{tabular}

\section{3信息流视角下金融集聚影响经济增长的实证分析}

本文根据理论假设 $\mathrm{H} 1$ 构建实证模型，如式（7）所示。

$$
E G_{\mathrm{it}}=\alpha+\beta_{1} F A_{\mathrm{it}}+\beta_{2}\left(F A_{\mathrm{it}} I H_{\mathrm{it}}\right)+\beta_{3}\left(F A_{\mathrm{it}} I T_{\mathrm{it}}\right)+\beta_{4} P C_{\mathrm{it}}+\beta_{5} H C_{\mathrm{it}}+\varepsilon_{\mathrm{it}}
$$

根据韩春庭（2003） ${ }^{[18]}$ 的观点，金融发展与经济增长之间存在内生性问题，因此本文在 进行实证分析时要考虑并解决内生性问题。参考李延凯和韩延春 (2013) ${ }^{[19]}$ 的实证研究, 本 文使用动态面板系统广义矩估计方法 (GMM) 消除内生性问题, 并进行实证分析, 结果如表 8 所示。

表8＼cjkstart信息流视角下金融集聚影响经济增长的GMM回归结果

\begin{tabular}{|c|r|r|r|r|}
\hline 变量 & 系数估计值 & \multicolumn{1}{|c|}{ 标准差 } & \multicolumn{1}{|c|}{ 统计量 } & \multicolumn{1}{c|}{$\mathbf{p}$ 值 } \\
\hline $\mathrm{EG}(-1)$ & -0.09 & 0.02 & -5.75 & 0.00 \\
\hline $\mathrm{FA}$ & -3.28 & 0.18 & -18.48 & 0.00 \\
\hline $\mathrm{FA} *^{*} \mathrm{H}$ & 0.82 & 0.05 & 16.95 & 0.00 \\
\hline $\mathrm{FA}$ IT & -0.43 & 0.06 & -6.66 & 0.00 \\
\hline $\mathrm{PC}$ & -0.60 & 0.03 & -19.59 & 0.00 \\
\hline $\mathrm{HC}$ & -1.11 & 0.15 & -7.23 & 0.00 \\
\hline $\mathrm{n}$ & 310 & Sargan 检验 $(\mathrm{p}$ 值 $)$ & 0.19 & \\
\hline
\end{tabular}

从上表可以看出, Sargan 检验的 $\mathrm{p}$ 值大于 0.1 , 无法拒绝 “模型过度约束正确” 的原假设, 即上述实证模型的过度约束正确, 实证结果可靠。式 (7) 的所有变量均通过了 $1 \%$ 显著性水 平下的假设检验, 但 EG(-1)、FA、FA*IT、PC、 HC 的系数估计值均为负数, 与本文提出的 理论假设 $\mathrm{H} 1$ 不符。只有 $\mathrm{FA}$ * IH 的系数估计值为正，符合本文提出的理论假设 $\mathrm{H} 1$ ：金融集聚 会带来信息流集聚, 信息流效应通过降低企业的交易成本和信息搜寻成本，从而促进经济增 长。 


\section{4金融创新视角下金融集聚影响经济增长的实证分析}

本文根据理论假设 $\mathrm{H} 2$ 构建实证模型, 如式 (8) 所示。

$$
E G_{\mathrm{it}}=\gamma+\varphi_{1} F A_{\mathrm{it}}+\varphi_{2}\left(F A_{\mathrm{it}} F I_{\mathrm{it}}\right)+\varphi_{3} P C_{\mathrm{it}}+\varphi_{4} H C_{\mathrm{it}}+\mu_{\mathrm{it}}
$$

实证结果如表 10 所示。

表9 金融创新视角下金融集聚影响经济增长的GMM回归结果

\begin{tabular}{|c|r|r|r|r|}
\hline 变量 & 系数估计值 & \multicolumn{1}{|c|}{ 标准差 } & t 统计量 & \multicolumn{1}{|c|}{$\mathbf{p}$ 值 } \\
\hline EG(-1) & -0.07 & 0.01 & -6.27 & 0.00 \\
\hline FA & -3.50 & 0.26 & -13.71 & 0.00 \\
\hline FA*FI & 0.28 & 0.02 & 12.20 & 0.00 \\
\hline PC & -0.67 & 0.04 & -18.93 & 0.00 \\
\hline HC & -0.79 & 0.14 & -5.53 & 0.00 \\
\hline $\mathrm{n}$ & 310 & Sargan 检验 $(\mathrm{p}$ 值 $)$ & 0.23 & \\
\hline
\end{tabular}

从上表可以看出, Sargan 检验的 $\mathrm{p}$ 值大于 0.1 , 无法拒绝 “模型过度约束正确” 的原假设, 即上述实证模型的过度约束正确, 实证结果可靠。式 (8) 的所有变量均通过了 $1 \%$ 显著性水 平下的假设检验, 但 $\mathrm{EG}(-1) 、 \mathrm{FA} 、 \mathrm{PC} 、 \mathrm{HC}$ 的系数估计值均为负数, 与本文提出的理论假设 $\mathrm{H} 1$ 不符。只有交叉项 FA*FI 的系数估计值为正, 符合本文提出的理论假设 H1: 金融集聚通 过知识溢出效应和区域创新网络效应提高区域金融创新速度, 金融创新提高了资本的边际生 产率、私人储蓄率和储蓄向投资的转化率，从而促进经济增长。

\section{4. 结论}

本文利用因子分析法测度区域金融集程度。论文实证结果表明我国各省份的金融集聚程 度非常不均匀，东部沿海地区的金融集聚程度高于中部地区的金融集聚程度，中部地区的金 融集聚程度又高于西部地区的金融集聚程度, 但各省份的金融集聚程度随着时间的推移整体 呈现缓慢提高趋势。

本文利用动态面板广义矩估计 (GMM) 方法进行回归分析。实证结果表明金融集聚程度、 信息腹地边界、信息厚度、金融创新等指标对区域经济增长的影响是显著的，但不同指标对 区域经济增长影响的方向不一致, 经济增长一阶滞后因子（EG(-1))、金融集聚程度（FA）、 实物资本 $(\mathrm{PC}) 、 人 力$ 资本 $(\mathrm{HC})$ 、金融集聚程度与信息厚度的交叉项 (FA*IT) 的回归系数 为负, 与本文所提出的理论假设不相符。只有金融集聚程度与信息腹地边界的交叉项 (FA*IH) 和金融集聚程度与金融创新的交叉项（FA*FI）的数估计值为正，符合本文提出的理论假设。

\section{References}

[1] Schumpeter J A, The Theory of Economic Development. Cambridge, MA:Harvard University Press, 1911.

[2] Goldsmith R.W,Financial Structure and Development, New Haven: Yale University Press, 1969.

[3] King R G, Levine R,Finance and growth:Schumpeter might be right, The quarterly journal of economics,108(3):717-737,1993.

[4] Levine R,Financial Development and Economic Growth:Views and Agenda,The Journal of Economic Literature, 35:42-78, 1997.

[5] Levine R,Finance and Growth: Theory and Evidence,Working paper,Mar18,2005. 
[6] Li Lin,Ding Yi,LiuZhihua,Spatial Econometric Analysis of the Effect of Financial Agglomeration on Regional Economic Growth Spillovers, Journal of Financial Research, 5 : 113-123, 2011.

[7] Guan Chunyan, Research on the Development of Financial Industry Based on Financial Perspective, Journal of Yuncheng University,31 (2): 35-38,2013.

[8] Xu Zhe, The Impact of Financial Agglomeration on Financial Industry: An Analysis of the Pathways and Effects,Journal of Business Economics, 26: 85-86,2015.

[9] Liu Jun,Huang Jieyu, Research on Financial Agglomeration Influencing Entity Economic Mechanism,Management World, (4):152-153,2007.

[10]Lu Jun,Xu Jie, An Empirical Analysis of Financial Agglomeration and Regional Economic Growth - A Case Study of Beijing - Tianjin - Hebei Region, Academic exchange,(2):15-21,2014.

[11]Pagano M,Financial Markets and Growth: An Overview ,European Economic Review,(2): 613-622, 1993.

[12]Porteous D J, The Geography of Finance:Spatial Dimensions of Intermediary Behaviour,Aldershot: Avebery, 1995.

[13]Porteous D J, The development of financial centers: location, information, externalities and path dependence,Money and the Space Economy. Chichester: Wiley,95-114,1999.

[14]Martin R.L,The New Economic Geography of Money,Money and the Space Economy, John Wiley\&Sons, Chichester, 1999.

[15] Thrift N,On the social and cultural determinants of international financial centers: the case of the City of London,Money, Power and Space,Oxford: Blackwell, 327-355,1994.

[16]Zhao Xiaobin, Wang Tan,Zhang Jinxi,Information flow and asymmetric information are determinants of financial and service center development: China case, Economic geography, (4): 408-414,2002.

[17]Zhao X B,Spatial Restructuring of Financial Centers in Mainland China and Hong Kong-A Geography of Finance Perspective,Urban Affairs Review ,38:35-571,2003.

[18]Han Chunting, The Endogenous Mechanism of Financial Development and Economic Growth,Journal of Tsinghua University (Philosophy and Social Sciences), 18(1):80-85,2003.

[19]Li Kaichun,Han Chunting, Financial Development and Economic Growth under the Evolution of Financial Environment: An International Experience,World Economy8:145-160,2013. 